\title{
Building Blocks for Information Literacy
}

\author{
By Judy Repman \& Randall Carlson \\ Georgia Southern University, GA
}

\begin{abstract}
Information Literacy includes a range of strategies and skills that are critical for educators in the $21^{\text {st }}$ century. This article focuses on a module developed to provide instruction related to one important set of Information Literacy skills: web searching. Key concepts relevant to basic web searching strategies, web searching tools and the use of pathfinder pages are defined and discussed. Examples of different tools and strategies are presented along with practice activities.
\end{abstract}

\section{Introduction}

The Information Age is part of the new reality of working with students in colleges of education. Students turn to the Web as their first (and often their only) information resource-they want their information full text, online, right now. Those of us who view the results of this searching often have serious doubts about this rush to accept the first item from the Yahoo! hit list as an authoritative resource. As college of education faculty members, we teach graduate and undergraduate education students introductory computer courses and graduate courses for school library media specialists in training.

When asked, most of our students assert that they have no trouble finding information on the Web. To try to gain attention, we then like to give our students a little challenge by asking them to find specific information on a simple topic (such as lesson plans on dinosaurs or resources on mainstreaming students). What usually follows are loud complaints that these items just don't exist on the Web.

Luckily, we believe that Web searching is a skill that can be taught and learned. We also believe that it is a critical building block of Information Literacy. We also realize, as Marylaine Block recently pointed out, that "while students might in fact wish to improve their searching skills, they very much doubt that we're going to be any help" (2001, p. 33) . In response to our perceptions of our students' Web searching skills, our knowledge of Information Literacy, and some understanding of our students' attitudes, we developed an instructional module that provides students with the kinds of knowledge and hands-on experiences with web searching tools and strategies that they can use immediately and apply in other settings.

\section{Information Literacy and Educators}

In Information Literacy Competency Standards for Higher Education (2000), the Association of College and Research Libraries points out that Information Literacy is a basic skill in our increasingly complex, technological world. With so many information sources available, the ability to locate, identify and use reliable sources is critical for an educated populace. Standard Two states that the "information literate student accesses needed information effectively and efficiently". Performance indicators for this standard include identification of appropriate information systems (or resources), developing effective search strategies, and retrieving the information.

Our module focuses on this particular area of Information Literacy for several reasons:

- Despite their assertions to the contrary, students are not effective web searchers.

- Use of a common introduction puts everybody "on the same page".

- Improved web searching brings immediate posi -tive results and helps students with their daily information needs across all of their classes.

-These instructional activities can easily be modified to match students' ability levels (since we occasionally have students with almost no prior web experience).

-The activities, resources listed, and examples used can be adapted for different subjects and different levels of knowledge about subjects (i.e., master's level special education majors, pre-service secondary social studies students or doc- 
toral level educational leadership students).

Despite their usefulness, web searching skills and strategies are rarely directly taught and practiced in other classes in a systematic way. Developing this module also gave us an opportunity to model a systematic approach to teaching Information Literacy that pre- and in-service educators can apply in their own classrooms. While Information Literacy is a critical skill for everyone, we firmly believe in this "ripple in the pond" impact when it comes to educators.

The remainder of this article describes a systematic web-searching module developed over the past five years. It is presented in its most general form and includes three components: web search basics, web search tools (directories, search engines, metasearch tools, kids search tools, and specialized search tools) and the use of pathfinders as an alternative to searching. The examples mentioned are those that we have found particularly useful. Given the rapidly changing nature of the web, we're always watching for something new and exciting to add to the module-we hope that you will do the same.

\section{Web Search Basics}

\section{Internet and Web Structure}

Understanding the structure of the Internet can be challenging. By defining the Internet defined as a worldwide network of interconnected computer systems and computer networks we demonstrate that searching for information may involve searching Many formats of electronic transfer. Email, bulletin boards, gopher, FTP (file transfer protocol) sites, discussion lists, or the World Wide Web are included in this array. The World Wide Web (WWW) is defined as a worldwide series of linked documents written using the protocol (computer language) called HTTP (hypertext transfer protocol). Further discussion focuses on the Web because it is the easiest place to find information and most of the time it proves to be an adequate source of information.

Today the Web can be separated into two parts, the Web as we usually consider it (sometimes called the "visible web") and the so-called "invisible web." We point out to our students that the visible web is composed of a large and rapidly growing set of linked web pages (some 3 billion is the current estimate; Sullivan a, 2001) that are "indelible" - that is, able to be cataloged by our current technologies and techniques. The invisible web, however, is much larger (estimated to be 500 times the size of the visible web) and is growing at a faster rate (Bergman, 2000). Invisible web information is readily available to those who have the skills and the inclination to find it. Why then, you may ask, do we concentrate on searching the visible web with our pre-service teachers? The answer goes back to our previous statementit is the easiest place to find information and most of the time it proves to be an adequate source of information.

\section{Basic Searching Strategies}

This area of our module has undergone some significant modifications over the past year. Previously, we introduced all of our students to Boolean logic and walked through several examples of constructing search strategies using Boolean operators. We still provide this information for our school library media students but we've come to the conclusion that for most general purpose search tools (described in the next sections) a simpler approach is often just as effective and is less confusing. Search Engine Math (2001) presents a simplified approach to Boolean logic, utilizing "+" and " - " to construct search statements.

One concept related to search engine math is another building block of Information Literacy: being able to specify the information needed in precise terms. We ask students to tell us what kinds of information needs they have using assignments from other classes. This is a very popular exercise since we use different search tools to demonstrate how the " + " and the " - " makes finding the information needed for actual assignments much more precise. The other special search strategy we demonstrate is the use of phrase searching, which most search tools recognize when quotation marks enclose the search phrase. These simple, yet powerful, strategies are easy for most students to remember . Students can see how much more effective their searches can be by the results they see.

\section{Web Search Tools}

Once students understand the structure of the WWW and general search basics, we highlight examples of the following general categories of search tools (all commonly misnamed "search engines"):

- Directories

- Search Engines

- Metasearch Tools

- Kid's Search Tools

- Specialized Search Tools

Use of any of these tools involves understanding one truth not evident to most of our students - that when they "search the Web" they are never searching the real-time web, but they are searching a database that has been constructed at a previous time to represent the web. The size of that database, the way that database was constructed, the way that the database is 
searched, and the way the search tool presents the results erns the choice of search tool. Even the largest database represents only a small portion of the Web. The most recent scientific study found that the largest database indexed only $16 \%$ of the total WWW and that the 10 largest databases only index about $42 \%$ of the Web (Lawrence \& Giles, 1999). Still, there are many useful search tools available for educators and our next step is to demonstrate these tools by category.

\section{Directories}

Directories are lists of information arranged in hierarchical categories. A person (who may or may not be a librarian) establishes the categories and their relationships. Directories are the most library-like in appearance and function. To use a directory, one must enter the category structure and then follow the hierarchy down to the final piece of information. Because editors review and categorize web pages for inclusion in the directory, directories can be small but focused. However, if your subject of interest is included, the results often provide a list of good, relevant sites.

Yahoo!, the most familiar directory, is composed of 14 top-level categories providing access to some $1.5 \mathrm{mil}-$ lion unique links (Sullivan b, 2001). Another very useful directory, the Librarians' Index to the Internet, contains more than 7,900 Internet resources selected and evaluated by librarians for their usefulness. Using the Librarians' Index to the Internet, it is easy to demonstrate the value of the annotations in selecting educational web sites. It is also useful to compare the clearly defined categories in the Librarian's Index with the overlapping and confusing category structure of Yahoo!.

\section{Search Engines}

Search engines construct their databases automatically using software programs called "spiders" or "bots" that "crawl" from one web page to another using the hyperlinks as a path. The size of the database clearly is an important factor. Another important factor is the algorithm that is used by the search engine to identify and rank returns for relevance. Each search engine is different and these characteristics frequently are not obvious, so finding a good search engine that works for you is important.

We suggest that our students try Google. It has the largest database (1.4 billion pages claimed as of July, 2001 ) and uses a relevancy algorithm called PageRank that analyzes links in terms of how many there are and who they are linked to. Yahoo! uses the Google database when searching for pages and not categories. FAST Search is our second choice for a search engine. It has the second largest database size and claims that it will grow with the Web - and it is fast! When time permits, or if our audience is more experienced, we show how these search engines can be personalized and what features are available for power searching. For students in advanced courses we also like to demonstrate the search features of Northern Light .

\section{Metasearch Tools}

Metasearch tools permit the simultaneous use of multiple search engines. They return a single, integrated and ranked set of results that is usually limited to 50-75 highly relevant items. We encourage our students to try Ixquick. Ixquick has a very clean interface and uses a very useful and intuitive relevance ranking. The site with most stars is ranked the highest .Each time a site is ranked in the top ten returns when the query is submitted to the ten individual search engines it receives a star. Ixquick also translates advanced search techniques to each search engine's syntax. Metacrawler's advantages include a customizable feature and access to the large Google database.

\section{Kid's Search Tools}

Kid's search tools are a category of search tools new to most of our students. Obviously, when searching for educational resources, it is important for teachers to be able to locate resources for student use as well as resources for their own use. Kid's search tools are an excellent way to show pre-service educators how to evaluate the reading level of a web page and to look at other factors like the depth of information included and the amount of advertising on a page.

$A L F Y$ is very popular with early childhood education students. ALFY has been developed for non-readers, so the categories are "read" when the mouse passes over the icons on the page. Even though the ALFY database is very small, it is an excellent tool to demonstrate how web resources can be identified for young users. KidsClick! is excellent for identifying resources for elementary aged students, while Searchopolis has sets of links for elementary, middle, and high school students. Whenever we give assignments we always try to differentiate between web resources that are appropriate for use by $\mathrm{K}-12$ students and those that teachers would use for information.

\section{Specialized Search Tools}

Specialized search tools can be defined as search tools that search for specific kinds of information, perhaps in a single subject or for information in specific formats. One need that our students have is to find graphics to use in preparing different assignments. We like to demonstrate Ditto, Google's Image Search, and 
AltaVista's Image Search as examples of specialized search tools. When a search term is entered, results are presented in terms of thumbnail graphics. This makes identifying an appropriate graphic much easier than the alternative of reading through and guessing from the text based results lists produced by general search tools. Other examples of specialized search tools include EduHound and EdView, which are limited to "educational" content, and FREE (Federal Resources for Educational Excellence) which allows searches for resources across governmental agencies.

\section{Pathfinders}

Pathfinders are the final category of search tools presented in the module. Usually, at this point students are suffering from information overload so they are usually relieved to hear that there is an alternative to searching. Pathfinders are resources developed and maintained by people with interest and expertise in a subject area. We believe that a "good" pathfinder is a well-organized list of links that is regularly maintained and is limited to a defined target audience. Probably the most familiar educational pathfinder is Kathy Schrock's Guide for Educators. Other examples of pathfinders include S.C.O.R.E. , which provides links related to children's and young adult literature and Gander Academy's Theme Related Resources on the World Wide Web .

Most state departments of education and major professional associations for educators now maintain similar links libraries that can be helpful pathfinders. We stress that pathfinders can be excellent starting points when information is needed. Our students usually love the idea that somebody else has done the work for them and they are amazed the there are people willing to do this amount of work just because they want to. Of course many faculty and education librarians have developed their own pathfinder pages so this is an excellent opportunity to point students to these "local" resources.

\section{Conclusion}

We provide our students with this Information Literacy framework and foundation through in-class lecture and demonstration. Even more important is how we follow through by providing distributed, targeted, hands-on practice. These two exercises have worked well for us and can be adapted to almost any kind of higher education setting.Students work in groups to look for different kinds of information using each of the search tools. We encourage students to use assignments from any of their classes for this activity. Students have to describe the strategies used and evaluate their results in terms of the usefulness of the information retrieved. Students choose a content area theme early in the term as a focus for all in-class activities. This theme is used as a search term. This provides ample opportunity to discuss the importance of refining search terms to improve the usefulness of the returns as they complete a variety of assignments. This is also useful in demonstrating the limitations of the web. By limiting their searching to a particular content area, we usually find that students begin to return to print resources for breadth and depth of coverage.

When referring to the art and science of Web searching, Paul Gilster (1997) notes that "ungoverned and perhaps ungovernable, the Internet's vast holdings catalyze your thinking only if you master the primary skills of the digitally literate searcher." (p. 3) Unfortunately, few students seem to realize their own limitations in terms of web searching skills and they may doubt that we can teach them anything about it. The instructional module presented here is simple, direct, and adaptable. Staff and faculty working in education libraries could partner with college of education faculty to build this component into classroom instruction and team teach the concepts. The time spent is well worth the results.

\section{References}

Bergman, M. (2000). The deep web: Surfacing hidden value. Brightplanet.com LLC . Retrieved on July 30, 2001 from http://www.brightplanet.com/ deep_content/index.asp.

Block, M. (2001). Teaching kids indirectly. Library Journal netconnect, (summer) 33-34.

Information Literacy Competency Standards for Higher Education. Retreived July 30, 2001 from http://www.ala.org/acrl/ilcomstan.html.

Gilster, P. (1997). Digital literacy. New York: Wiley Computer Publishing.

Lawrence, S. \& Giles, L. (1999). Accessibility of information on the web. Nature, 400, 107-109.

Search Engine Math. (2001).Retreived July 30, 2001 from http://searchenginewatch.com/facts/math.html

Sullivan, D. (a).(2001). Search engine sizes. The Search Engine Report, April 6, 2001. Retrieved July 7, 2001 from http://searchenginewatch.com/ reports/sizes.html

Sullivan, D. ( b ).(2001). Search engine sizes, The Search Engine Report, Retrieved April 6, 2001 from http://searchenginewatch.com/reports/directories.html

Judy Repman \& Randall Carleson are affiliated with The College of Education, Georgia Southern University, Statesboro, GA

E-mail:jrepman@gasou.edu

E-mail:rcarlson@gasou.edu 